\title{
Fairly direct hit! Advances in imaging of shotgun projectiles in MRI
}

\author{
Sebastian Eggert • Rahel A. Kubik-Huch • Markus Klarhöfer • \\ Alexander Peters • Stephan A. Bolliger • Michael J. Thali • \\ Suzanne Anderson • Johannes M. Froehlich
}

Received: 12 August 2014 / Revised: 22 December 2014 / Accepted: 30 January 2015 / Published online: 25 February 2015

(C) The Author(s) 2015. This article is published with open access at Springerlink.com

\begin{abstract}
Objectives To investigate the magnetic properties of different types of projectiles and qualify the metal artefact reduction technique for diagnostic and/or forensic MRI.

Materials and methods Ten different projectiles embedded in ordnance gelatine blocks underwent an in vitro 1.5-T MR study with seven sequences including a recently developed metal artefact reduction sequence (Advanced WARP) combining VAT (view-angle-tilting) and SEMAC (slice-encoding metal-artefact-correction). Resulting image quality (five-point scale: $1=$ best; $5=$ worst) was scored. Quantifiable magnetic characteristics were correlated with qualitative rating of the MR sequences and torque dislodgment.

Results Metal artefact reduction sequence (median: 2.5$)$ significantly $(\mathrm{p}<0.001)$ improves depiction of projectiles in comparison to all other MR pulse sequences (median: 4.75). Images from diamagnetic composed bullets (median: 2) are much less disturbed compared to magnetic attracted ones (median: 5). Correlation (0.623) between deflection angle
\end{abstract}

S. Eggert $(\bowtie) \cdot$ R. A. Kubik-Huch · A. Peters $\cdot$ S. Anderson Department of Radiology, Kantonsspital Baden AG, Im Ergel, 5404 Baden, Switzerland

e-mail: sebastian.eggert@irm.uzh.ch

M. Klarhöfer

Siemens Healthcare, Zurich, Switzerland

S. Eggert $\cdot$ S. A. Bolliger $\cdot$ M. J. Thali

Institute of Forensic Medicine, University of Zurich,

Zurich, Switzerland

S. Anderson

Radiology, Sydney School of Medicine, University of Notre Dame

Australia, Sydney, NSW, Australia

J. M. Froehlich

Federal Institute of Technology, Pharmaceutical Sciences,

Zurich, Switzerland measurement (ferromagnetic mean $84.2^{\circ}$; paramagnetic $62^{\circ}$; diamagnetic mean $0^{\circ}$ ) and median qualitative image quality was highly significant $(p=0.027)$. Torque dislodgement was distinct for elongated magnetic attracted projectiles.

Conclusions Significant improvement of MR imaging of projectiles using metal artefact reduction techniques has important implications for diagnostic/forensic work-up. The correlations between magnetic attraction force, deflection-angle results and image properties demonstrate that the MR safety of projectiles can be estimated with one of these methods.

Key Points

- Metal artefact reduction sequence improves overall image quality of bullets $(p<0.001)$.

- Deflection angle assessment significantly predicts image quality of bullets ( $p=0.027$ ).

- Classification of projectiles' magnetic properties based on artefacts' characteristics is possible.

- Classifying of bullets has important implications in diagnostic and forensic imaging.

- Identification of projectiles' magnetic attributes improves estimation of patients' injury risk.

Keywords Magnetic resonance imaging $\cdot$ Safety $\cdot$ Artefacts · Forensic sciences $\cdot$ SEMAC

\section{Introduction}

Imaging shotgun injuries in patients can be challenging in both the clinical emergency setting as well as for forensic interpretation in the deceased.

Computed tomography (CT) is a widely used technology in clinical practice and more recently also in forensic medicine, to detect and localise remaining foreign bodies, e.g. bullets [1-5]. State-of-the art CT systems, with optimised imaging parameters and software-based algorithms can reduce 
artefacts in diagnostic $\mathrm{CT}$ to improve depiction and thus identification of foreign bodies [6].

Magnetic resonance imaging (MRI) bears the advantage of better soft tissue contrast. However, potential ferromagnetic properties of the foreign body that may endanger the patient, in particular translational attraction and/or torque causing movement and dislodgement need to be considered. Moreover, in the deceased, forensic interpretation might be constrained due to secondary movement of the remaining bullet potentially inducing damage to the surrounding tissue.

From the diagnostic point of view metal artefacts originating from the bullets remain a serious problem in MRI, hampering image interpretation. Adjacent soft tissues may be distorted by strong local off-resonances, due to the highly variable susceptibility constants of metals. The magnetic properties of the bullets, the amount of ferromagnetic metals, their specific shape, the surrounding tissue, the strength of the static magnetic field and the spatial distribution of the gradient magnetic field have an influence on field distortions and may lead to a loss of diagnostic information [7, 8]. New metal artefact reduction techniques such as a combination of VAT (view-angle tilting), SEMAC (slice-encoding metal artefact correction), high-bandwidth RF pulses, high readout gradient amplitude and fat suppression techniques are promising. VAT reduces in-plane artefacts, whereas SEMAC addresses through-plane misregistrations. These different techniques ('Advanced WARP' and clinically available 'WARP', Siemens Healthcare, Erlangen, Germany) have been implemented recently on clinical MR scanners to reduce through-plane as well as in-plane susceptibility artefacts [9].

Moreover, there have been several investigations regarding safety of metallic objects in MRI [10-12]. One of the key references for MRI safety issues is the Shellock's MRI safety website [13] referencing a large number of objects, including several ballistic items. While inherent dangers have been addressed, the question of how to overcome the imaging challenges of metallic projectiles remains.

Thus the aim of the present study was twofold, firstly to characterise the magnetic properties of different types of projectiles commonly encountered in ordnance, sporting, hunting or crime-related usage in a 1.5-T MRI environment, and secondly to assess the potential of new MR metal artefact reduction technique applied to these different projectiles.

\section{Materials and methods}

In vitro experiments and phantoms

The prospectively investigated projectile types were selected according to the following criteria:
1) Exemplary of those commonly encountered in ordnance, sporting, hunting or crime-related usage: for example, the 9-mm Luger as former ordnance and currently frequently used sporting bullet is also found in crime-related cases. The $7.62-\mathrm{mm}$ projectile (.308 Winchester) is in use in NATO armies or for long-distance hunting.

2) To cover a broad range of susceptibility properties (ferro-, para- or diamagnetic composition).

3) To expand safety data [13].

The following projectiles were thus included: a $2.5-\mathrm{mm}$ steel pellet (declared as ferromagnetic); full metal jacket (steel compounded) projectiles of $9 \mathrm{~mm}$ (.354 Luger), $7.62 \mathrm{~mm}$ (.308 NATO), $7.5 \mathrm{~mm}$ (GP 11 Swiss), $11.43 \mathrm{~mm}$ (.45 ACP) and $5.56 \mathrm{~mm}$ (.223 Remington, NATO), each with lead cores. A special projectile of $7.62 \mathrm{~mm}$ (.308 Winchester, Armor Piercing, RUAG) with a tombac (brass alloy with high copper content and zinc adjunction) jacket and tungsten carbide core supposed to be paramagnetic was also included. Ultimately, projectiles of $4.5 \mathrm{~mm}$ composed of pure lead (.177 'wasp waist'), projectiles of $9 \mathrm{~mm}$ (.357 Magnum) and of $11 \mathrm{~mm}$ (.44 Magnum) both with copper semijacket and lead core (all declared as diamagnetic) were chosen (Fig. 1).

Each projectile was embedded in the centre of a $10 \%$ ballistic gelatine (Gelita AG, Eberbach, Germany) 1.5-L block hardened in a plastic bucket, as described previously [14]. Additionally we placed six lipophilic nitroglycerine capsules (Streuli Pharma AG, Uznach, Switzerland) on the external surface serving as a localisation reference. These phantoms with a cross-diameter of $18 \mathrm{~cm}$ were stored in a refrigerator and finally imaged after passively adapting to room temperatures between 16 and $20^{\circ} \mathrm{C}$.

\section{MR imaging}

MR images were acquired using a 1.5-Tesla MRI system (MAGNETOM Aera - software version: syngo MR D13, Siemens Healthcare, Erlangen, Germany) with a 20-channel receive-only volume head-coil. The phantoms underwent MR scanning in coronal and axial plane with the following imaging techniques using conventional clinical pulse sequences: T1-weighted spin-echo (T1-SE; repetition time (TR) $500 \mathrm{~ms}$, echo time (TE) $8.9 \mathrm{~ms}$ ), proton-density weighted turbo-spinecho (PD-TSE; TR $2500 \mathrm{~ms}$, TE $4.6 \mathrm{~ms}$, turbo-factor 15), T2weighted turbo-spin-echo (T2-TSE; TR $3000 \mathrm{~ms}$, TE $88 \mathrm{~ms}$, turbo-factor 16), ultrashort echo-time turbo-spin-echo (UTE; TR $7.91 \mathrm{~ms}$, TE $0.06 \mathrm{~ms}$ ), T2*-weighted gradient-echo (T2FL2D; TR $507 \mathrm{~ms}$, TE $25 \mathrm{~ms}$, flip angle (FA) $20^{\circ}$ ), gradient echo imaging for magnetic field mapping (GRE; TR $400 \mathrm{~ms}$, TE $2.7 \mathrm{~ms}$ and $7.5 \mathrm{~ms}, \mathrm{FA} 55^{\circ}$ ) and a TSE sequence for metal artefact reduction combining the VAT and SEMAC techniques (WARP). VAT and SEMAC were used as implemented in the research package 'Advanced WARP'. 


\begin{tabular}{|c|c|c|c|c|c|c|c|c|c|}
\hline $\begin{array}{l}7.5 \mathrm{~mm} \text { GP } \\
11\end{array}$ & $\begin{array}{l}5.56 \mathrm{~mm} \\
\text { NATO }\end{array}$ & 9mm Luger & $\begin{array}{l}7.62 \mathrm{~mm} \\
\text { NATO }\end{array}$ & $\begin{array}{l}.357 \\
\text { Magnum }\end{array}$ & .44 Magnum & $.45 \mathrm{ACP}$ & $\begin{array}{l}4.5 \mathrm{~mm} \text { lead } \\
\text { projectile }\end{array}$ & $\begin{array}{l}7.62 \mathrm{~mm} \\
\text { RUAG AP }\end{array}$ & $\begin{array}{l}2.5 \mathrm{~mm} \text { steel } \\
\text { pellet }\end{array}$ \\
\hline 春 & 春 & 車 & $\equiv$ & 音 & 青 & 春 & 拝 & 无 & 无 \\
\hline 三 & 青 & 春 & 春 & 春 & 春 & 春 & 春 & 春 & 春 \\
\hline 莑 & 春 & 春 & 奉 & 春 & 春 & 恶 & 春 & 拝 & 春 \\
\hline 春 & 春 & 青 & 严 & 无 & 春 & 恶 & 春 & 弄 & 春 \\
\hline 刍 & 春 & 奉 & 羔 & 春 & 春 & 春 & 春 & 刍 & 春 \\
\hline 无 & 青 & 彗 & 奉 & 青 & 堷 & 春 & 刍 & 春 & 青 \\
\hline & & & & & & & $\bar{E}^{\prime \prime \prime}$ & 春 & 春 \\
\hline
\end{tabular}

Fig. 1 To-scale images of all investigated projectiles. Distance between single bars corresponds to $1 \mathrm{~mm}$ on both axes

The relevant pulse sequence parameters of the Advanced WARP package were: coronal acquisition, voxel size, $1.0 \times$ $1.0 \times 3.0 \mathrm{~mm}^{3}$; field of view, $256 \mathrm{~mm} \times 256 \mathrm{~mm}$; slices per scan, 24; TR, $2800 \mathrm{~ms}$; TE, $6.3 \mathrm{~ms}$; FA, 180; readout bandwidth, $558 \mathrm{~Hz} /$ pixel; turbo factor, 15; echo spacing, $6.26 \mathrm{~ms}$; parallel imaging technique, GRAPPA; acceleration factor, 2; SEMAC factor, 12; view-angle tilting; acquisition time, $5 \mathrm{~min} 42 \mathrm{~s}$.

\section{Magnetic field interactions}

\section{Deflection-angle quantification}

Similar to previous studies [12], the deflection-angle measurement was performed at 1.5-T to check for the influence of the static magnetic field on the bullets. A small plastic container was attached at the end of a $25-\mathrm{cm}$ long filament (own weight $0.9449 \mathrm{~g}$ ) that was fixed anteriorly in the middle of the upper cover of the head coil (Fig. 2). As deflection axis, a pole was installed in a horizontal direction at $10.5 \mathrm{~cm}$ beyond the upper fixing of the filament. Projectiles were placed carefully into the container and slowly moved with the head coil on the MR table into the centre of the gantry defined by the laser light cross. At this position the deflection-angle was measured with a non-ferromagnetic goniometer with $2^{\circ}$ graduated markings. Deflection from the vertical axis $\left(=0^{\circ}\right)$ was measured three times in a row and mean values were calculated.

\section{Translational magnetic attraction}

Translation magnetic attraction forces were quantified using a non-ferromagnetic spring scale (D.B.G.M, Germany) with $1 \%$ precision. The maximum force was analysed using the same container as described above with the respective projectile placed near the gantry at the 12 o'clock position. This was repeated three times in order to improve measurement accuracy and precision.

\section{Torque dislodgement force}

The torque dislodgement force when moving the object through the gantry was perceived by two observers holding each projectile in one hand moving it through the three axes at the gantry margin. Perceived force to move against flipping was rated qualitatively on a three-point scale $(0=$ no force; $1=$ weak torque force leading to reorientation; $2=$ strong torque dislodgement forcing reorientation).

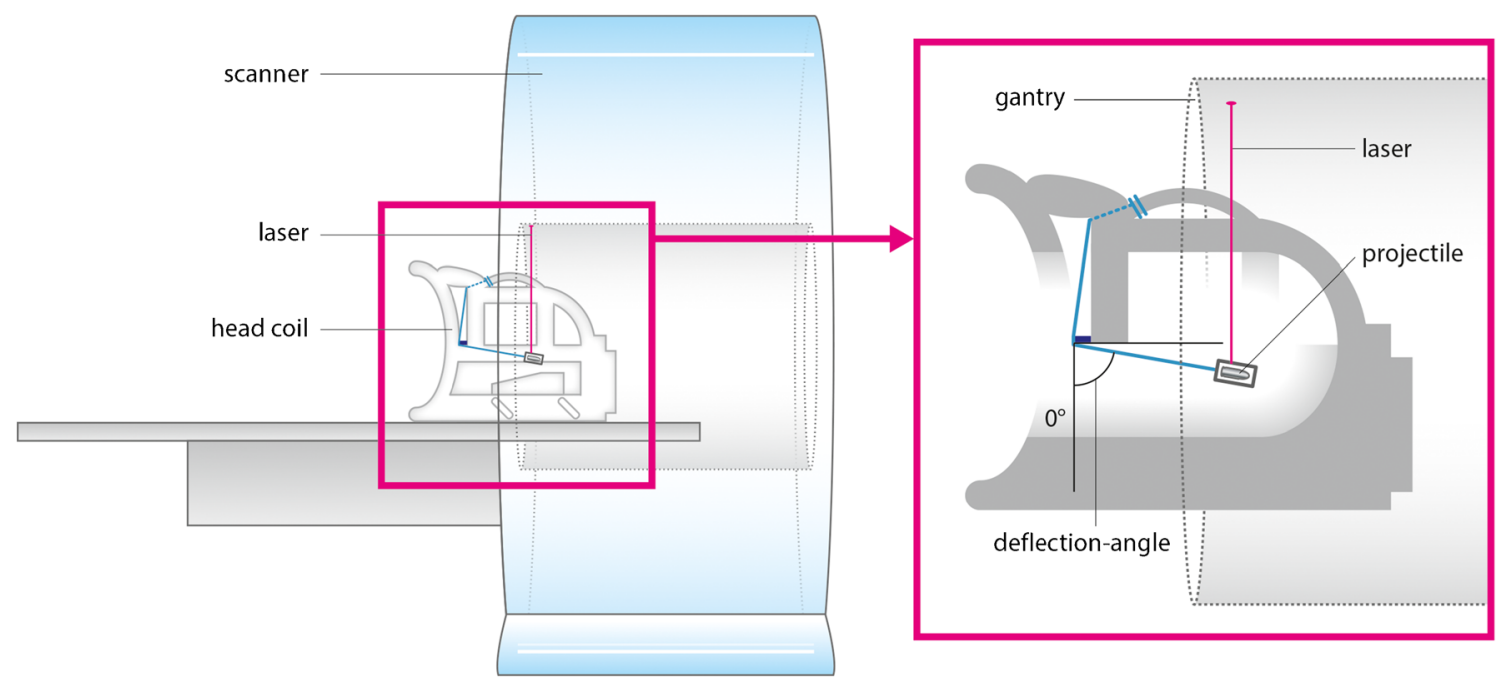

Fig. 2 Schematic representation of the deflection-angle measurement experiment at the gantry of the scanner. Illustration: Wolfgang Herzig - www.des-illu.de 
Image interpretation

Acquired MR-data were transferred to a separate workstation (syngo.via, Siemens Healthcare) for further qualitative and quantitative analyses using an integrated measuring device. MRI analyses were performed on the original acquired coronal or axial images choosing the image with maximum inplane depiction of the projectile or artefact.

Artefact size and resulting image quality of MRI were scored in consensus by two experienced readers (initials blinded for review; 3 and 7 years of clinical reading, respectively) using a five-point scale. Criteria ranging from best $=1$ up to worst $=5$ are described in detail in Figs. 2 and 3.

\section{Magnetic field mapping}

Magnetic field maps were calculated in Matlab (R2014a (version 8.3), The Mathworks, Natick, MA, USA) from gradient-echo images with echo times of $4.76 \mathrm{~ms}$ and $9.52 \mathrm{~ms}$ (GRE). For quantification, magnetic field gradients covering a measurement range of $15 \mathrm{~mm}$ each starting from two distances ( $15 \mathrm{~mm}$ and $45 \mathrm{~mm}$ ) apart from the bullet were calculated.

\section{Statistical analysis}

The exact Friedman test and Wilcoxon-Nemenyi-McDonaldThompson post-hoc tests were used for qualitative ratings of MR pulse sequences. The Kruskal-Wallis test and the Nemenyi-Damico-Wolfe-Dunn post-hoc tests were used to evaluate whether the median ratings for all MR pulse sequences differed between the ten projectiles. Kendall's tau $\tau$ was computed to determine the correlation between the deflection angle and the median rating of all MR sequences. Hypotheses were tested at a significance level of $p=0.05$. All analyses were performed using SPSS (version 20 and R software version 2.15.2 (package 'coin'); R Foundation for Statistical Computing, Vienna, Austria) [15].

\section{Results}

Qualitative evaluation of the MR images

The detailed qualitative evaluation of the MR images is given in Fig. 2. Susceptibility artefacts are much less disturbing on the images of diamagnetic-composed projectiles. Consequently qualitative grading in general is significantly better in all acquired sequences for these

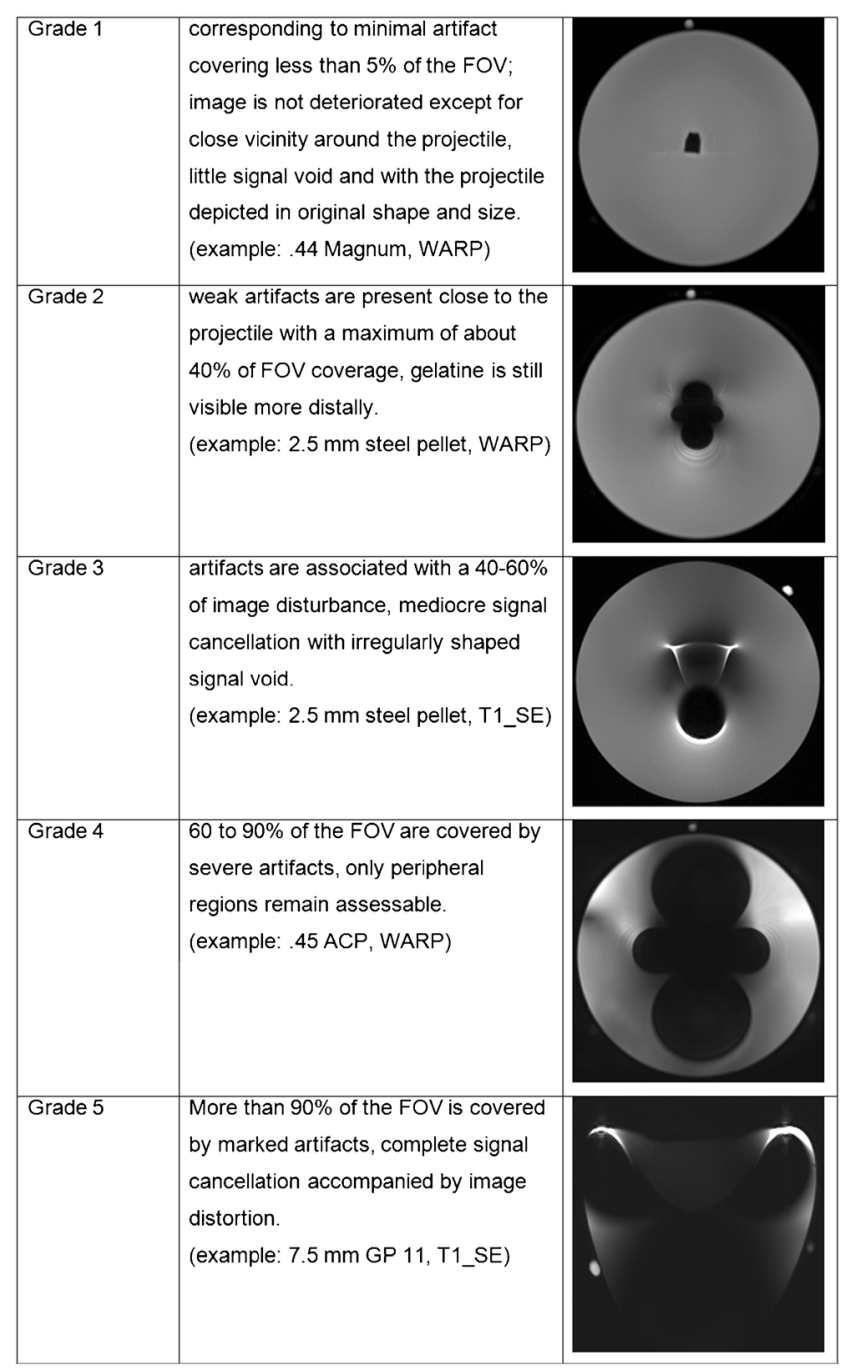

Fig. 3 Image quality assessment by two readers (in consensus) with typical MR image examples on a five-point scale: qualitative scoring and typical image samples. Note exemplary dipole shape of the ferromagnetic/paramagnetic-composed bullets on Grade 2 and 4 images. A regular and symmetrical dipole artefact shape is only encountered in case of strongly enhanced magnetisation when using Advanced WARP algorithm in the present setting

objects (median $=2$ ) in contrast to a worse median rating of the magnetic attracted projectiles (median 5) (Table 1, Fig. 3).

An additional finding is the generation of symmetrical dipole-shaped signal void artefacts using the WARP sequence only visible for bullets declared as having ferro- or paramagnetic composition (Fig. 4).

The exact Friedman test indicated that the seven MR sequences were significantly different with respect to their ability to depict the projectiles $(p<0.001)$. Post-hoc tests revealed that WARP images depicted projectiles significantly better than all other T1- $(p=0.004), T 2-(p=0.001)$ and protondensity $(p=0.027)$ weighted pulse sequences. 
Table 1 Qualitative ratings of MR sequences by two readers in consensus on a five-point scale with 1=best and 5=worst as described in detail in Fig. 3

\begin{tabular}{|c|c|c|c|c|c|c|c|c|}
\hline \multirow[t]{2}{*}{ Projectile } & \multicolumn{8}{|c|}{ MR sequence } \\
\hline & UTE & WARP & PD_TSE & GRE & T2-FL2D & T2_TSE & T1_SE & Median Rating \\
\hline 7.5-mm GP 11 & 4 & 3 & 5 & 5 & 5 & 5 & 5 & 5 \\
\hline 5.56-mm NATO & 3 & 2 & 4 & 4 & 5 & 5 & 5 & 4 \\
\hline 9-mm Luger & 4 & 3 & 5 & 5 & 5 & 5 & 5 & 5 \\
\hline 7.62-mm NATO & 4 & 3 & 5 & 5 & 5 & 5 & 5 & 5 \\
\hline .357 Magnum & 2 & 1 & 2 & 2 & 2 & 2 & 2 & 2 \\
\hline .44 Magnum & 2 & 1 & 2 & 1 & 3 & 3 & 1 & 2 \\
\hline $.45 \mathrm{ACP}$ & 5 & 5 & 5 & 5 & 5 & 5 & 5 & 5 \\
\hline $4.5-\mathrm{mm}$ lead projectile & 1 & 1 & 1 & 2 & 2 & 2 & 2 & 2 \\
\hline 7.62-mm RUAG AP & 4 & 3 & 4 & 5 & 5 & 5 & 5 & 5 \\
\hline 2.5-mm steel pellet & 3 & 2 & 3 & 3 & 5 & 3 & 4 & 3 \\
\hline Median rating & 3.5 & 2.5 & 4.0 & 4.5 & 5 & 5 & 5 & \\
\hline
\end{tabular}

Comparison of projectiles and image quality

The Kruskal-Wallis test indicated that the image quality differed between the ten projectiles. Post-hoc tests showed that .357 Magnum, .44 Magnum and 4.5-mm lead projectiles are significantly $(\mathrm{p}<0.001)$ more depictable than $7.5 \mathrm{~mm}$ GP 11 , $5.56 \mathrm{~mm}$ NATO, $9 \mathrm{~mm}$ Luger, $7.62 \mathrm{~mm}$ NATO, .45 ACP and $7.62 \mathrm{~mm}$ RUAG AP.
Deflection-angle quantification and spring-scale attraction force

Descriptive data of the single projectiles, their deflectionangle and spring-scale attraction force are given in Table 2. As expected, the magnetic-attracted objects present a higher deflection angle (ferromagnetic declared projectiles: mean $84.2^{\circ}$; range $78-88^{\circ} ; 62^{\circ}$ for the paramagnetic bullet) from

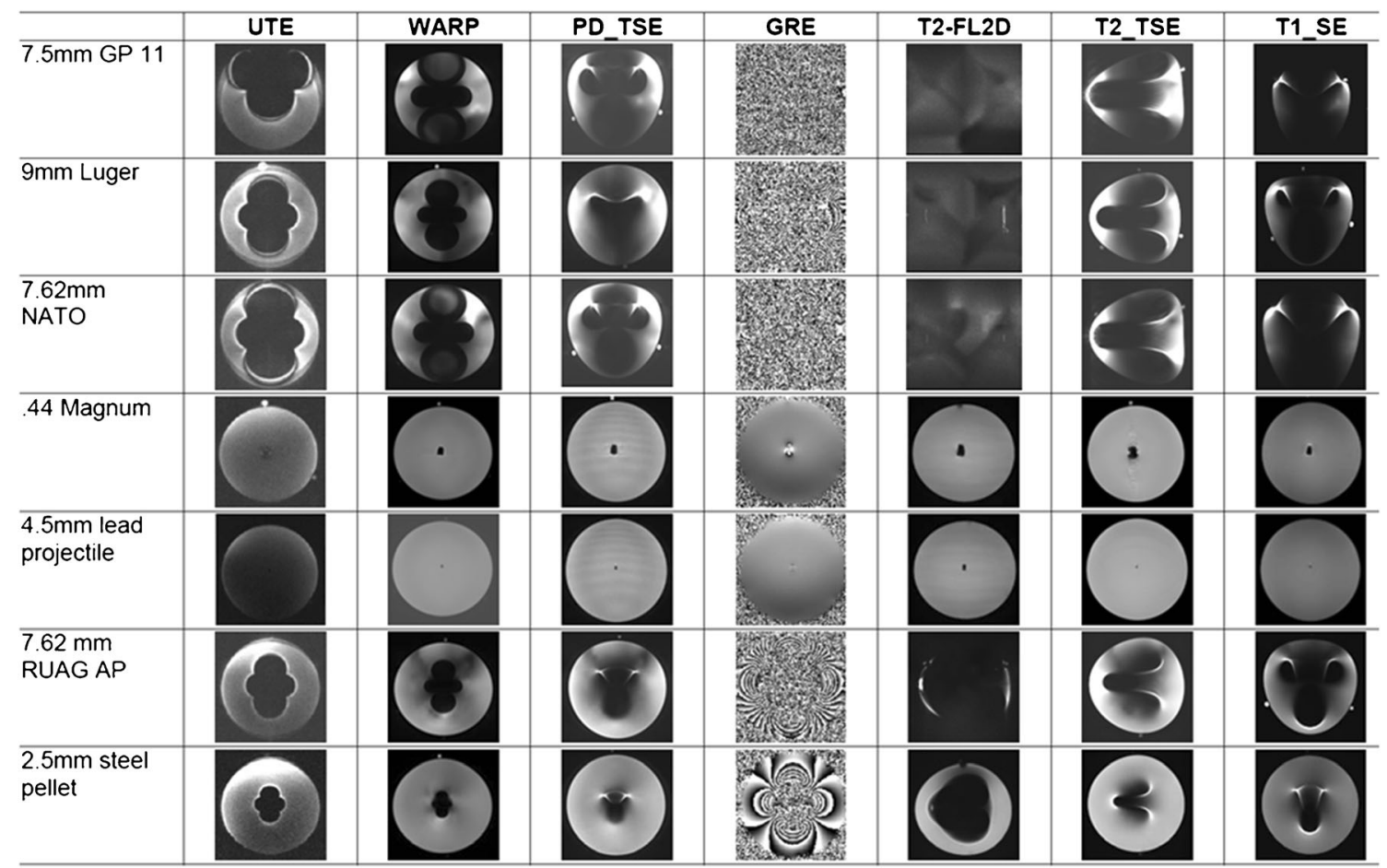

Fig. 4 Examples of various projectiles and their depiction on different MR pulse sequences (WARP here corresponds to Advanced WARP). The represented field of vision (FOV) covers the entire phantom on the coronal plane. GRE images correspond to the calculated field maps performed on gradient echo images 


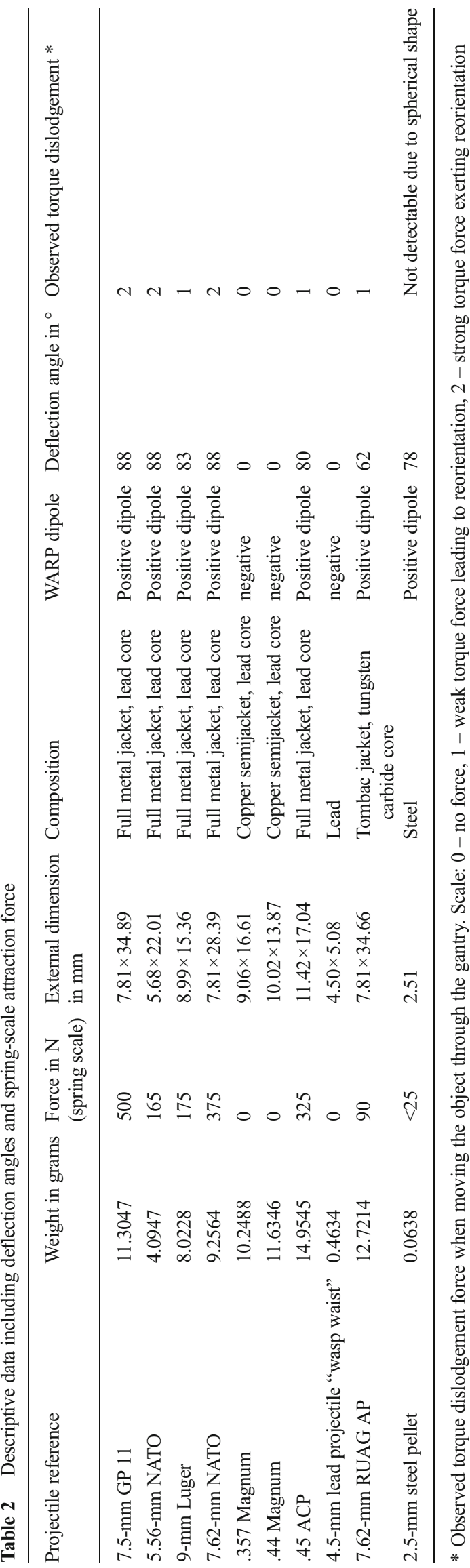

the vertical axis than those with an absence of strong magnetic attraction $\left(\right.$ mean $\left.0^{\circ}\right)$. Similar results were yielded from the spring-scale magnetic attraction force measurements with some slight weight-dependent differences.

Correlation of deflection-angle with MR image quality

Kendall's tau $(\tau=0.623)$ indicated that increased deflectionangle values significantly correlate with worse image quality ratings $(\mathrm{p}=0.027)$.

Torque dislodgement force

During translational spring force measurements distinct dislodgement and torque movement of the ferro- and paramagnetic-composed bullets was observed. Systematic qualitative rating revealed that elongated projectiles with magnetic attraction had a strong tendency to torque and reorientate themselves along the static magnetic field (mean $=1.75$; Table 2).

MRI safety aspect

Overall, based on our observations, we consider the following in the MRIsafety.com database not-listed projectiles as unsafe in MRI: 7.5-mm GP 11, 5.56-mm NATO, 9-mm Luger (Swiss made), 7.62-mm RUAG AP and 2.5-mm steel pellet (Table 3). However, the $4.5-\mathrm{mm}$ lead 'wasp waist' not-listed projectile is rated as MRI safe based on our results. For the remaining four projectiles (7.62-mm NATO, .357 Magnum, .44 Magnum, .45 ACP; see Table 3) previous ratings could be confirmed [13].

Field map analysis

The magnetic-attracted bullets lead to strong signal voids in the gradient echo images used for magnetic field mapping. Therefore, magnetic field maps could only be calculated for the smallest ferromagnetic projectile and the diamagnetic ones (Table 4). Results show that even on the potential diamagnetic projectiles magnetic field changes were quantifiable. The local field gradient was stronger closer to the bullet. As expected, the relatively small ferromagnetic steel pellet $(2.5 \mathrm{~mm})$ yielded more elevated field changes compared to the diamagnetic objects.

\section{Discussion}

In the presence of projectiles the tested metal artefact reduction pulse sequence (Advanced WARP) recently implemented on clinical MRI scanners significantly improves image quality compared to other conventional pulse sequences. The significant correlation between magnetic attraction force, 
Table 3 Tabular MRI safety comparison of the assessed projectiles taking in account the MRISafety.com website [13] recommendations together with our results

\begin{tabular}{llll}
\hline Bullet type/reference & Listed on MRIsafty.com & MRIsafty.com recommendation & Our safety rating \\
\hline 7.5-mm GP 11 & Not listed & - & Unsafe \\
5.56-mm NATO & Not listed & - & Unsafe \\
9-mm Luger (Swiss made) & Not listed & - & Unsafe \\
7.62-mm NATO & Listed & Unsafe 1 & Unsafe \\
.357 Magnum & Listed & Safe & Safe \\
.44 Magnum & Listed & Safe & Safe \\
.45 ACP & Listed & Unsafe 1 & Unsafe \\
4.5-mm lead projectile & Not listed & - & Safe \\
7.62-mm RUAG AP & Not listed & - & Unsafe \\
2.5-mm steel pellet & Not listed & - & Unsafe \\
\hline
\end{tabular}

deflection-angle results and image artefact properties demonstrate that the MR safety of projectiles can be estimated with either of these methods.

The 'Advanced WARP' pulse sequence specifically developed to reduce susceptibility artefacts in comparison to routinely used MR sequences has proven to be effective in prostheses $[16,17]$. This could be confirmed herewith for projectiles. Moreover, 'Advanced WARP' according to our results is more effective in reducing metal artefacts even in comparison to conventional MR techniques suggested in case of disturbing foreign bodies with increased susceptibility (using shortening of TE, avoiding gradient-echo pulse sequences or optimising bandwidth [18-20]).

In clinical routine as well as in a forensic setting, reduction of image distortion and of artefact size are important features allowing inclusion of persons reporting the presence of projectiles that may potentially disturb the MR image quality [21]. In cases where projectiles are located in less damageable tissue or already surrounded by scar, distant from sensitive tissue, suspension of an MRI examination might thus be avoided. Nevertheless, artefacts with signal voids close to magnetic projectiles are still present on Advanced WARP images, though they are much smaller in the case of nonferromagnetic objects. As shown in the present study the magnitude of the artefacts correlates with the magnetic susceptibility of the composition and components of the bullets, but is also influenced by the bullets' shape, size and sequence parameters [12]. Furthermore, if a projectile or remnant metal foreign body is found incidentally during MRI, our study supports that Advanced WARP or other sequences help classify the magnetic properties of the unknown foreign object (dipoles, artefact shape and size). Metal artefact reduction sequences like the advanced WARP package in case of low susceptibility objects may enable an early identification of bullet type (associated with specific gun usage) on the MR images. This may allow for early identification of suspected aggressors, potentially resulting in cost, time and manpower savings with reduction of official investigations at an earlier phase.

While the presence of dipole-shaped artefacts in MRI based on metallic properties has been addressed for dental foreign objects [22], no such artefact generation is addressed in publications regarding projectiles. The Advanced WARP technique leads to a clear and symmetrical depiction of the dipole-shaped signal voids since they are usually not overlaid by misregistered signals from other slice positions. If large dipoles with signal void are generated this suggests the presence of a ferromagnetic object. In the absence of larger artefacts, magnetic attraction or repulsion can be essentially neglected; however, possible heating effects or conductivity might still need to be considered. In contrast, recent publications showed only limited heating (max. $1.7^{\circ} \mathrm{C}$ at $3.0-\mathrm{T}$ ), which might be related to the limited size of projectiles [12].

Asymmetrical objects not oriented perpendicularly to the static magnetic field are potentially more in danger to be displaced or torque dislodged when moving close to the
Table 4 Field-map analyses of all measurable bullets exploited on GRE sequence

\begin{tabular}{lll}
\hline Bullets & \multicolumn{2}{l}{ Measurement ranges $(15 \mathrm{~mm}$ each) } \\
\cline { 2 - 3 } & $\begin{array}{l}\text { Range 1 starting at } 15 \mathrm{~mm} \\
\text { from projectile }(15-30 \mathrm{~mm})\end{array}$ & $\begin{array}{l}\text { Range 2 starting at 45 mm } \\
\text { from projectile (45-60 mm) }\end{array}$ \\
\hline $4.5-\mathrm{mm}$ lead projectile & $0.18 \mu \mathrm{T} / \mathrm{m}$ & $0.11 \mu \mathrm{T} / \mathrm{m}$ \\
9-mm (.357 Magnum) & $0.18 \mu \mathrm{T} / \mathrm{m}$ & $0.11 \mu \mathrm{T} / \mathrm{m}$ \\
11-mm (.44 Magnum) & $0.44 \mu \mathrm{T} / \mathrm{m}$ & $0.18 \mu \mathrm{T} / \mathrm{m}$ \\
$2.5-\mathrm{mm}$ steel pellet & Not conclusive due to signal void & $7.8 \mu \mathrm{T} / \mathrm{m}$ \\
\hline
\end{tabular}


gantry of the magnet, emphasizing the importance of preventive measures [23]. This is not always easy to ascertain as ferromagnetic detection systems are rarely installed (e.g. Ferroguard Screener; www.Metrasens.com). Ultimately, if the presence of ferromagnetic foreign bodies cannot be excluded, the benefit-risk ratio of MRI examinations must be carefully evaluated [24]. Our results, especially with the torque dislodgement, emphasize once more that the MR safety of ferromagnetic projectiles remains an important concern [23]. While slight pressure on adjunct tissue or dislodgement of bullets might be acceptable or controllable in a clinical environment, this should be judged more critically in forensic cases. Any secondary change potentially could modify forensic interpretation leading to miscarriage of justice.

Several of the studied bullets have not been previously listed in the extensive MRI safety.com database [13]. Therefore, our results might be used to extend this database. It should be emphasized that the tungsten carbide core projectile with paramagnetic declaration could not be differentiated from steel containing ferromagnetic samples on our MR images, justifying its classification as 'unsafe' despite slightly reduced magnetic attraction. Paramagnetic projectiles are not listed separately in the database [13]. Last but not least, MR safety classification of single projectiles might differ from country to country. The 9-mm exists in a US variant classified as 'safe' [10,13], while a Swedish type, as well as the Swiss type included here (Luger), are defined as 'unsafe' [10,13].

Advanced WARP or similar metallic artefact reduction techniques may help the radiologist and the forensic pathologist in diagnosing and follow-up imaging of recurrent tumour, fracture, infection, bleeding or other pathologies/changes in the region of ferromagnetic bullets or objects with similar attributes. Besides projectiles, implants or prostheses, there are numerous other devices such as surgical clips, dental fillings, fixation screws, metallic stents or surgical pins and meshes, and all kinds of electronic devices that on their own can complicate imaging techniques [19]. The rising number of interventions, reconstruction techniques and future technical developments with the use of miniaturised devices support the need for such artefact reduction methods and their evaluation.

\section{Limitations}

This study had several limitations. Firstly, this phantom study included a relatively small number of projectiles, weakening the statistical power. Our study objects, however, cover a broad range of various types of projectiles with differing magnetic properties. The majority of these are still not characterised for MRI. Secondly, the chosen projectiles had not been used previously in guns, thus not presenting deformations or other alterations. In practice, heterogeneously deformed or even fragmented projectiles would be expected. Such modified bullets or fragments keep their magnetic properties, potentially inducing further susceptibility. In addition, increased tissue damage due to sharpness following deformation or shape modification cannot be ruled out.

Thirdly, other manufacturers of MRI systems developed similar susceptibility artefact reduction methods such as MARS (Philips Healthcare, Amsterdam, The Netherlands) or MAVRIC / MAVRIC-SL (GE Healthcare Systems, Little Chalfont, UK). Our results are specific for the 'Advanced WARP' technique comprising SEMAC and VAT at 1.5-T (Magnetom Aera, Siemens Healthcare, Erlangen, Germany) and might differ from those from other vendors.

Finally, projectiles were not scanned within injured persons or dead bodies for ethical reasons, thus needing further validation.

\section{Conclusions}

In summary, in the setting of projectiles, metal artefact reduction techniques such as the 'Advanced WARP' package result in a statistically significant improvement in MR image quality compared to conventional pulse sequences.

Potentially better image quality translates to improved clinical diagnostic and forensic results. Despite improved susceptibility artefact reduction methods specific risks such as torque dislodgement or magnetic attraction need to be considered when imaging bullets.

Acknowledgments We thank Nicole Tracy Graf Johansen, statistician (www.biostatistics.ch), Winterthur, Switzerland for the statistical analysis, and Wolfgang Herzig, dipl.-Designer (www.des-illu.de), Essen, Germany for the schematic illustration (see Fig. 2). The scientific guarantor of this publication is Johannes M. Froehlich. The authors of this manuscript declare relationships with the following companies: Markus Klarhoefer: Author is an employee of Siemens Healthcare and supports external research projects such as this one; however, there was not influence on resulting data or interpretation. Johannes M. Froehlich: Author is a consultant of Guerbet contrast media company. Financial activities related to the present article: none to disclose. Other relationships: Invitations from Philips Healthcare and Siemens Healthcare to present on User meetings with payment of travel expenses. Consultant for Primex Pharmaceuticals. The authors state that this work has not received any funding. Institutional Review Board approval was not required because the study was a phantom study. Methodology: experimental, performed at one institution.

Open Access This article is distributed under the terms of the Creative Commons Attribution Noncommercial License which permits any noncommercial use, distribution, and reproduction in any medium, provided the original author(s) and the source are credited.

\section{References}

1. Thali MJ, Viner MD, Brogdon BG (eds) (2011) Brogdon's forensic radiology, 2nd edn. CRC press, Boca Raton 
2. Wullenweber R, Schneider V, Grumme T (1977) A computertomographical examination of cranial bullet wounds. Z Rechtsmed 80:227-246

3. Thali MJ, Yen K, Schweitzer W, Vock P et al (2003) Virtopsy, a new imaging horizon in forensic pathology: virtual autopsy by postmortem multislice computed tomography (MSCT) and magnetic resonance imaging (MRI): a feasibility study. J Forensic Sci 48:386-403

4. Yen K, Vock P, Christe A et al (2007) Clinical forensic radiology in strangulation victims: forensic expertise based on magnetic resonance imaging (MRI) findings. Int J Legal Med 121:115-123

5. Ross S, Ebner L, Flach P et al (2012) Postmortem whole-body MRI in traumatic causes of death. AJR Am J Roentgenol 199:1186-1192

6. Winklhofer S, Benninger E, Spross C et al (2014) CT metal artifact reduction for internal fixation of the proximal humerus: value of mono-energetic extrapolation from dual-energy and iterative reconstructions. Clin Radiol 69:e199-e206

7. Zimmermann BH, Faul DD (1984) Artifacts and hazards in NMR imaging due to metal implants and cardiac pacemakers. Diagn Imaging Clin Med 53:53-56

8. New PF, Rosen BR, Brady TJ et al (1983) Potential hazards and artifacts of ferromagnetic and nonferromagnetic surgical and dental materials and devices in nuclear magnetic resonance imaging. Radiology 147:139-148

9. Advanced WARP - Features \& Benefits - Siemens Healthcare Global. https://www.healthcare.siemens.com/magnetic-resonanceimaging/options-and-upgrades/clinical-applications/advanced-warp/ features. Accessed 14 April 2014

10. Teitelbaum GP, Yee CA, VanHorn DD, Kim HS, Colletti PM (1990) Metallic ballistic fragments: imaging safety and artifacts. Radiology 175:855-859

11. Hess U, Harms J, Schneider A, Schleef M, Ganter C, Hannig C (2000) Assessment of gunshot bullet injuries with the use of magnetic resonance imaging. J Trauma 49:704-709

12. Dedini RD, Karacozoff AM, Shellock FG, Xu D, McClellan RT, Pekmezci M (2013) MRI issues for ballistic objects: information obtained at 1.5-, 3- and 7-Tesla. Spine J 13:815-822
13. MRISafety.com, Shellock R \& D Services, Inc. and Frank G. Shellock, PhD, http://www.mrisafety.com/TheList_search.asp. Accessed 14 April 2014

14. Coupland R, Rothschild M, Thali M, Kneubuehl B (eds) (2011) Wound ballistics. Springer Medizin, Berlin

15. Hothorn T, Hornik K, van de Wiel MA, Zeileis A (2006) A lego system for conditional inference. Am Stat 60:257-263

16. Ai T, Padua A, Goerner F et al (2012) SEMAC-VAT and MSVATSPACE sequence strategies for metal artifact reduction in $1.5 \mathrm{~T}$ magnetic resonance imaging. Investig Radiol 47:267-276

17. Sutter R, Ulbrich EJ, Jellus V, Nittka M, Pfirrmann CW (2012) Reduction of metal artifacts in patients with total hip arthroplasty with slice-encoding metal artifact correction and view-angle tilting MR imaging. Radiology 265:204-214

18. Kolind SH, MacKay AL, Munk PL, Xiang QS (2004) Quantitative evaluation of metal artifact reduction techniques. J Magn Reson Imaging 20:487-495

19. Hargreaves BA, Worters PW, Pauly KB, Pauly JM, Koch KM, Gold GE (2011) Metal-induced artifacts in MRI. AJR Am J Roentgenol 197:547-555

20. Lee MJ, Kim S, Lee SA et al (2007) Overcoming artifacts from metallic orthopedic implants at high-field-strength MR imaging and multi-detector CT. Radiographics 27:791-803

21. Dewey M, Schink T, Dewey CF (2007) Frequency of referral of patients with safety-related contraindications to magnetic resonance imaging. Eur J Radiol 63:124-127

22. Starčuk Z, Bartušek K, Hubálková H, Bachorec T, Starčuková J, Krupa P (2006) Evaluation of MRI artifacts caused by metallic dental implants and classification of the dental materials in use. Meas Sci Rev 6:357-362

23. Shellock FG, Crues JV (1988) High-field-strength MR imaging and metallic biomedical implants: an ex vivo evaluation of deflection forces. AJR Am J Roentgenol 151:389-392

24. Karacozoff A, Pekmezci M, Shellock F (2013) Armor-piercing bullet: 3-T MRI findings and identification by a ferromagnetic detection system. Mil Med 178:380-385 